Milena Doytcheva, Politiques de la diversité. Sociologie des discriminations et des politiques antidiscriminatoires au travail

Bruxelles, PIE Peter Lang, coll. « Travail \& Société », 2015

Vincent-Arnaud Chappe

URL : http://journals.openedition.org/travailemploi/7319

DOI : 10.4000/travailemploi.7319

ISSN : 1775-416X

Éditeur

DARES - Ministère du Travail

Édition imprimée

Date de publication : 1 octobre 2016

Pagination : 106-109

ISSN : 0224-4365

Référence électronique

Vincent-Arnaud Chappe, « Milena Doytcheva, Politiques de la diversité. Sociologie des discriminations et des politiques antidiscriminatoires au travail », Travail et Emploi [En ligne], 148 | octobre-décembre 2016, mis en ligne le 11 juillet 2019, consulté le 25 septembre 2020. URL : http://journals.openedition.org/ travailemploi/7319; DOI : https://doi.org/10.4000/travailemploi.7319 


\title{
Politiques de la diversité. Sociologie des discriminations et des politiques antidiscriminatoires au travail
}

\author{
Milena Doytcheva
}

Bruxelles, PIE Peter Lang, coll. «Travail \& Société », 2015, 196 p.

\section{Lu par Vincent-Arnaud Chappe*}

L'ouvrage Politiques de la diversité. Sociologie des discriminations et des politiques antidiscriminatoires au travail est tiré de l'habilitation à diriger des recherches de Milena Doytcheva. Il s'inscrit dans une trajectoire de recherche portant sur les questions d'ethnicité et de discrimination, appréhendées notamment à travers ce qu'en disent les politiques publiques françaises. L'ouvrage propose d'apporter une analyse empiriquement fondée sur l'émergence de la thématique de la "diversité » dans l'espace public à partir des années 2000, et plus particulièrement dans le monde de l'entreprise. L'auteure s'interroge donc sur les conditions d'appropriation d'une notion « allogène », issue notamment de l'histoire américaine des droits civiques, dans le contexte spécifique d'une France républicaine porteuse dans les discours - si ce n'est toujours en pratique - de valeurs universalistes et volontiers « aveugles à la couleur » (colour-blind). Que devient le mot d'ordre de la diversité dans un pays où l'indifférenciation et la neutralisation des identités dans l'espace public font régulièrement figure d'idéal ? Comment la diversité s'articule-t-elle avec les exigences juridiques d'égalité qui découlent du principe de non-discrimination ? La diversité peut-elle également pointer vers un horizon inclusif et de reconnaissance ? À moins qu'elle ne soit que le paravent d'une idéologie néolibérale et dépolitisante, hostile aux identités collectives, voire « nationaliste » et assimilationniste ? C'est à ces différentes questions que s'attelle la recherche de l'auteure, dans une perspective à la fois diachronique et centrée sur les usages effectifs de la notion de diversité.

L'ouvrage se divise en trois parties, comprenant chacune trois ou quatre chapitres. La première partie est consacrée à la genèse à la fois des politiques de lutte contre les discriminations et de celles promouvant la diversité en France, et met notamment en évidence l'ancrage entrepreneurial de ces dernières. Elle revient en particulier sur l'idée que les politiques de la diversité peuvent être comprises, sur le modèle étasunien,

\footnotetext{
* Centre de sociologie de l'innovation - Institut interdisciplinaire de l'innovation (CSI-i3), CNRS, Mines ParisTech, PSL Research University.
} 
comme la traduction des exigences du droit de la non-discrimination dans la logique managériale : il y aurait dans le cas français une disjonction entre ces deux logiques qui seraient de plus autonomes. La diversité ne se contente pas de retraduire le droit dans un vocabulaire positif mais obéit à sa propre dynamique.

La deuxième partie s'intéresse aux logiques de la diversité telles qu'elles sont portées par les politiques d'entreprise. Elle montre d'abord que les rationalités qui y sont attachées sont extra-juridiques et mettent surtout en avant des arguments méritocratiques ${ }^{1}$, alors revisités, moraux ou économiques ${ }^{2}$. Elle informe également du processus d'extension du champ de la diversité, et de hiérarchisation des « critères » liés. Dans ce mouvement, l'ethnicité, à l'origine du mot d'ordre de la diversité, se trouve paradoxalement marginalisée, euphémisée, voire niée.

La troisième partie est probablement la plus intéressante et la plus innovante : elle se focalise sur le rôle joué par les « intermédiaires » et « opérateurs » de la diversité, c'est-à-dire les structures, principalement associatives, qui viennent en aide aux entreprises dans la mise en œuvre des politiques se réclamant de ce principe. Financées principalement par l'argent public, elles déploient des programmes d' accompagnement et de placement de demandeurs d'emploi, souvent situés à la frontière de la légalité, notamment parce qu'ils ciblent des populations minoritaires repérées en fonction de critères ethniques que le droit ne devrait pas leur permettre d'appréhender. Chargés de ces activités qu'ils ne peuvent que difficilement assumer, ces intermédiaires se retrouvent également en situation, selon l'analyse de M. Doytcheva, de coproduire des pratiques potentiellement discriminantes en soutenant un discours fondé sur les idées d'employabilité, de compétences et de savoir-être dont ces populations minoritaires seraient toujours soupçonnées d'être en déficit. Ce travail d'accompagnement verrait également se répandre un "usage sélectif de la diversité », à des fins normalisatrices et assimilationnistes : il exclurait par exemple l'expression d'appartenances et de pratiques religieuses minoritaires telles que le port du voile pour les femmes musulmanes. Face à ces pratiques fortement prescriptives, les populations accompagnées réagissent de différentes manières traduisant leur degré variable de prise de conscience du phénomène. Les positions diverses qu'elles prennent rappellent celles des associations d'accompagnement et de promotion de la diversité (entre minimisation, prise en compte préventive et affirmation du caractère systémique de la discrimination).

Cette recherche nous permet d'entrer en profondeur dans la fabrique française des politiques de diversité. Elle nous montre, à partir de terrains variés et d'analyses poussées, les ambiguïtés et les impensés de ce mot d'ordre. Ce faisant, l'auteure jette un éclairage pertinent et très critique sur l'appropriation nationale de cet horizon potentiellement inclusif. On appréciera notamment la démonstration portant sur l'action de ces intermédiaires à qui est déléguée l'action de faire sans dire et qui, contraints

1. Par exemple la possibilité ouverte à chacun de décrocher un emploi dans l'entreprise en fonction de ses compétences.

2. De tels arguments avancent par exemple l'idée d'un lien entre diversité et performance. 
de contourner les restrictions du cadre légal républicain, ne peuvent légitimer publiquement leur action.

Quelques critiques peuvent néanmoins être livrées. La première tient à la forme narrative, qui ne permet pas toujours de restituer avec précision le travail d'enquête. L'écriture est très dense, mais n'offre que peu de précisions sur les démarches empiriques qui ont présidé à la collection et à l'analyse des résultats. L'auteure utilise par exemple beaucoup l'expression « l'enquête montre » mais sans pour autant ouvrir la boîte noire du protocole de recherche.

Sur le fond de l'analyse, on peut également reprocher à M. Doytcheva de ne pas mobiliser davantage de cadres théoriques autres que celui de la sociologie des discriminations. L'analyse aurait gagné à exploiter plus directement les concepts de la sociologie économique, de la sociologie de l'action publique ou de la sociologie des professions par exemple. Le néo-institutionnalisme sociologique aurait ainsi permis d'appréhender encore plus finement la diffusion du mot d'ordre de diversité, ou le découplage entre affichage symbolique de valeurs inclusives et pratiques réelles. Dans la même optique, il aurait été possible de s'appuyer davantage sur les travaux de Lauren Edelman ${ }^{3}$ et sur son analyse de la mise à distance du droit dans les politiques de diversité (même si l'on a bien compris que la situation française ne peut être calquée sur celle des États-Unis).

Une dernière critique, enfin, porte sur le rapport à une posture d'analyse compréhensive : si l'approche critique est bien sûr parfaitement justifiée, elle opacifie parfois les raisons d'agir des acteurs de la diversité. Surtout, elle ne permet pas de mettre suffisamment en valeur les tensions qui structurent néanmoins l'espace d'intervention en matière de diversité, tensions que l'auteure souligne pourtant. Un exemple nous paraît caractéristique : celui du droit. La thèse de l'oubli du droit nous semble en effet trop peu nuancée. Si une mise à distance de la légalité est indéniable, d'autres forces de rappel jouent dans le sens contraire : production d'une jurisprudence en matière de discrimination concernant au moins certains critères, mais également rôle joué par certaines organisations plus légalistes que d'autres, au premier rang desquelles la Haute Autorité de lutte contre les discriminations et pour l'égalité (Halde), devenue depuis 2011 le Défenseur des droits. Les entreprises n'ont pas le pouvoir de « décréter » l'oubli du droit : cette éviction nécessiterait des conditions sociales très particulières qui expliqueraient une totale ineffectivité de la forme juridique. Or ces conditions ne nous semblent pas réunies ici, ce qui explique d'ailleurs qu'une partie du travail de « diversité » passe par des formations au droit de la non-discrimination. Sans postuler pour autant une influence forte de ce dernier, on pourrait ainsi s'interroger sur la façon dont la diversité participe à façonner la légalité - comprise comme l'ensemble émergeant d'interactions sociales orientées par la conscience du droit - plutôt qu'elle ne vienne s'y substituer. L'enquête pourrait alors être prolongée vers d'autres acteurs

3. Edelman L. B. (2011), «L'endogénéité du droit », in Bessy C., Delpeuch T., Pélisse J. (dir.), Droit et régulations des activités économiques : perspectives sociologiques et institutionnalistes, Paris, LGDJ-Lextenso, pp. 85-109. 
et dispositifs «frontières », en contact plus direct avec le discours juridique et ses équipements formels, à l'instar de la direction des ressources humaines ou des affaires juridiques. Ces critiques sont donc une invitation pour le champ de recherche sur les discriminations à continuer le travail entrepris par Milena Doytcheva en approfondissant les résultats très novateurs de son enquête dense et empiriquement très riche. 